\title{
Myeloid cell biology and inhibition of anti-tumor immune responses by MPDL3280A in urothelial bladder cancer
}

\author{
Yuanyuan Xiao ${ }^{1 *}$, Christina Rabe ${ }^{2}$, Marcin Kowanetz ${ }^{1}$, Thomas Powles ${ }^{3}$, Nicholas J Vogelzang ${ }^{4}$, Daniel P Petrylak ${ }^{5}$, \\ Yohann Loriot ${ }^{6}$, Mitchell Denker ${ }^{1}$, Rin Nakamura ${ }^{1}$, Qun J Wu' ${ }^{1}$, Teiko Sumiyoshi ${ }^{1}$, Zachary Boyd ${ }^{1}$, \\ Siew-leng M Teng ${ }^{1}$, Xiaodong Shen'1, Gregg Fine', Daniel S Chen ${ }^{1}$, Priti S Hegde ${ }^{1}$
}

From Society for Immunotherapy of Cancer 29th Annual Meeting

National Harbor, MD, USA. 6-9 November 2014

Treatment options for metastatic urothelial bladder cancer (UBC) are limited. Mutational complexity is known to be high in UBC and may correlate with increased immunogenicity. MPDL3280A, a human PD-L1 monoclonal antibody containing an engineered Fc-domain designed to promote a Th1-driven response, has demonstrated a RECIST response rate of $43 \%$ in diagnostically selected, pretreated patients with UBC. A total of 68 patients (67 with efficacy evaluable) were enrolled in the UBC cohort of the Phase I study; $45 \%$ were PD-L1 IHC diagnostic positive as defined by expression of PDL1 on $\geq 5 \%$ of tumor-infiltrating immune cells. In the prescreened UBC population, the prevalence of PD-L1positive patients was $27 \%$.

Comprehensive gene expression analyses of UBC tumors were conducted to interrogate the tumor immune microenvironment in PD-L1-positive tumors and to identify potential mechanisms associated with response or resistance to MPDL3280A. In this study, PD-L1-positive tumors exhibited a high prevalence of gene expression markers associated with T-effector cells (Teff), including perforin, IFN $\gamma, \mathrm{CD} 8 \mathrm{~A}$, granzyme $\mathrm{B}$, granzyme A and EOMES. Additionally, a low baseline signature of genes associated with myeloid cell markers, including $I L 1 B$ and $I L 8$, appeared to be statistically significantly associated $(P<0.01)$ with MPDL3280A response, suggesting a potential role for myeloid biology in resistance to MPDL3280A treatment in UBC.

Tumor burden markers, including CA-125, CA19-9 and human chorionic gonadotropin (HCG), have been associated with chemotherapy response markers in UBC. A marked decrease in these markers, including CEA, CA19-9, CA-125 and HCG, was observed with MPDL3280A response after 1 treatment cycle, potentially enabling an on-treatment monitoring alternative for response to therapy. Similarly, evaluation of cytokines on treatment identified markers, including IL-6 and IL-10, elevated as early as Cycle 2 only in patients without response to MPDL3280A. These circulating cytokines and tumor-associated gene signatures suggest potential mechanisms associated with resistance and response to MPDL3280A in UBC and provide a rationale for informed combination strategies to further improve treatment benefit in this indication.

\section{Authors' details}

${ }^{1}$ Genentech, Inc., San Francisco, CA, USA. ${ }^{2}$ Roche Diagnostics GmbH, Germany. ${ }^{3}$ Barts Cancer Institute, Queen Mary University of London, London, UK. ${ }^{4}$ University of Nevada School of Medicine and US Oncology/ Comprehensive Cancer Centers of Nevada, NV, USA. ${ }^{5}$ Yale Cancer Center, New Haven, CT, USA. 'G Gustave Roussy, Villejuif, France.

Published: 6 November 2014

doi:10.1186/2051-1426-2-S3-P131

Cite this article as: Xiao et al:: Myeloid cell biology and inhibition of anti-tumor immune responses by MPDL3280A in urothelial bladder cancer. Journal for ImmunoTherapy of Cancer 2014 2(Suppl 3):P131.

${ }^{1}$ Genentech, Inc., San Francisco, CA, USA

Full list of author information is available at the end of the article 